\title{
CRE Recombinase
}

National Cancer Institute

\section{Source}

National Cancer Institute. CRE Recombinase. NCI Thesaurus. Code C17285.

CRE Recombinase catalyses site-specific recombination between two 34 base pair loxp sites and maintains the phage genome as a monomeric unit-copy plasmid in the lysog enic state. CRE is a homotetramer when bound to DNA and belongs to the phage integrase family. (from SWISS-PROT P06956 and NCI) 